\title{
Hyoid excursion during the swallow in stroke survivors
}

\author{
Youngsun Kim¹, Margaret A. Dietz ${ }^{1}$ Taeok Park ${ }^{2}$ \\ ${ }^{1}$ Communication Sciences and Disorders, Ohio University, Athens, Ohio; ${ }^{2}$ Communication Sciences and Disorders, Illinois State University, Normal, IL, \\ USA
}

Purpose: The purpose of the investigation was to examine whether duration, distance or velocity of hyoid excursion was associated with aspiration in post-stroke survivors.

Methods: Duration, distance and velocity of hyoid excursion were calculated using videofluoroscopic swallowing examinations (VFSEs) of 39 post-stroke survivors. Statistical comparisons were made using a two-way repeated measure analysis of variance (ANOVA) with between subject variable being aspirators and non-aspirators, and within subject variable being bolus volume ( $5 \mathrm{~mL}$ and $10 \mathrm{~mL}$ ). The significance level was set as $p<0.05$.

Results: The duration of hyoid excursion in aspirators was significantly slower than in nonaspirators. There were no significant differences in hyoid velocity or distance between aspirators and non-aspirators.

Conclusions: Slower hyoid excursion duration may put stroke survivors at risk of aspiration. It is necessary for the clinician to design the intervention strategies to compensate for or rehabilitate from slow hyoid excursion in stroke survivors.

Keywords: Swallowing, Dysphagia, Aspiration, Hyoid excursion, Stroke

\section{INTRODUCTION}

The process of swallowing consists of sequences of important physiological events that move food or liquid from the mouth to the stomach. Swallowing disorder, dysphagia can lead to aspiration pneumonia, malnutrition, dehydration, and even death [1]. About 10 million Americans are evaluated each year with swallowing difficulties [2]. Dysphagia prevalence increases with age and poses particular problems in older individuals. Approximately $7 \%$ to $10 \%$ of adults older than 50 years have dysphagia, although this number may be low because many patients with this problem do not seek medical care [3].

Oropharyngeal swallowing is divided into two phases: the oral phase and the pharyngeal phase [4]. The pharyngeal phase is considered involuntary, and once triggered, it continues as a fixed set of actions [5]. During the pharyngeal phase, several events occur quickly and almost simultaneously to move the bolus swiftly through the pharynx and into the esophagus while at the same time protecting the airway which is crucial to avoid aspiration. The hyoid and larynx move up and forward as a result of contraction of extrinsic tongue muscles that are attached to the hyoid [1]. As the hyoid moves, the larynx is pulled along with it due to its muscular connections. This is known as hy-

\section{(«ㄷㄷㄷㅁ}

Received: April 27, 2019

Revision: October 7, 2019

Accepted: December 7, 2019

\section{Correspondence:}

Youngsun Kim

Communication Sciences and Disorders, Ohio University, W233 Grover Center,

Athens, OH 45701-2979, USA

Tel: 1-740-597-1286

Fax: 1-740-593-0287

E-mail:kimy2@ohio.edu
(C) 2019 The Korean Association of SpeechLanguage Pathologists

This is an Open Access article distributed under the terms of the Creative Commons Attribution NonCommercial License (http://creativecommons.org/ licenses/by-nc/4.0/) which permits unrestricted noncommercial use, distribution, and reproduction in any medium, provided the original work is properly cited. 
olaryngeal excursion. Closure of the larynx is needed to form a seal to the entrance of the trachea in order to protect the respiratory airways. This closure is achieved through hyolaryngeal excursion. Another purpose of hyoid movement is the opening of the esophagus. This event allows the bolus to pass through the upper esophageal sphincter (UES) and into the esophagus, thus decreasing the chance of it becoming remnant in the pharynx and spilling into the larynx. This passage is crucial to avoiding aspiration as well. The muscles and nerves involved in mastication and swallowing heavily rely on signals from the brain to function sequentially. These signals direct the effort of the muscles of mastication to generate a bolus suitable for swallowing [6]. As the swallowing advances through the phases, the nerve signals then trigger the involuntary events such as the closing of the epiglottis and opening of the UES. A disruption in these steps can have serious medical implications, leading to dysphagia, aspiration, and possibly death.

According to the National Stroke Association (2000), strokes are the leading cause of dysphagia. Ischemic or hemorrhagic stroke results in a lack of blood flow and a buildup of blood that puts too much pressure on the brain, causing damage [7]. Because swallowing is such a complicated and involved process of the central nervous system, a stroke causing brain injury can heavily impact this process. The cause of dysphagia (e.g., aspiration) in stroke survivors is mainly related to the changes in physiology. In normal swallowing, vertical and anterior displacement of the hyoid occurs as the bolus passes into the pharynx [8]. Kim and McCullough [8] suggested that the vertical displacement of the hyoid is more associated with the closure and protection of the larynx, and anterior displacement is more associated with the opening of the UES. The combination of these movements decreases the risk of aspiration because the airway is completely sealed and protected by the epiglottis, while the UES is completely opened.

After a stroke, reduced maximal hyoid excursion has been suspected as one of the primary physiologic indicators of aspiration [9]. Maximal anterior displacement of the hyoid decreases, but maximal vertical displacement does not. However, there were no statistically significant differences in both maximum vertical and anterior displacement of the hyoid between aspirators and non-aspirators in stroke survivors. Kim and McCullough [9] proposed to investigate how the hyoid excursion is associated with the timing of aspiration, particularly before or during the swallow, but that there has been limited research done on the association between timing of aspiration and swallowing physiology. In addition, it is important to investigate the whole physiological process of hyoid excursion. This lack of research led us to examine the duration, distance, and velocity of hyoid excursion in two groups of stroke survivors, aspirators and non-aspirators. The analysis of hyoid excursion will help differentiate whether slow or fast hyolaryngeal excursion is associated with aspiration. If duration, distance, or velocity is in fact linked with aspiration, the data will help clinicians to examine stroke survivors at risk of aspiration, thus allowing for preventative measures such as diet restrictions, feeding alternatives, and consistency changes.

The objective of the study was to examine whether there was a difference in the duration, distance, or velocity of hyoid movement during the swallow in stroke survivors who did or did not aspirate. There have been limited studies measuring or exploring the velocity of hyoid excursion in disordered swallowing, particularly post-stroke swallowing. The outcomes of this study will provide the clinical information to find ways to avoid aspiration and related complications.

\section{METHODS}

\section{Subjects}

Videofluoroscopic swallowing examinations (VFSEs) of 39 stroke survivors were submitted for the analysis from the previous investigation [10]. The mean age of stroke survivors was 67.8 years and the mean number of days post-onset was 5.98 . Brain imaging revealed 39 stroke survivors suffered a single stroke at frontal, temporal or parietal lob in the cerebral cortex. The subjects were divided into two groups based on the presence of aspiration: Stroke survivors with aspiration $(N=10$ aspirators) and without aspiration ( $N=29$ non-aspirators). The occurrences of aspiration were before or after the swallow for aspirators.

\section{Videofluoroscopic Swallowing Examination (VFSE)}

The VFSE data was originally collected using a mobile, C-arm x-ray (OEC Diasonics, model 7600) system. Each subjects' swallows were recorded while they were sitting in an upright position in a stretcher chair. Videos were recorded onto a Panasonic Super VHS PV-S7670 Pro Line Multiplex videocassette recorder. The fluoroscopic tube was placed on the side of the subject to capture an image of the subject that included the lips on one side of the frame to the back of the neck on the other, and the nasopharynx superiorly to just below the UES area inferiorly. The two $5 \mathrm{~mL}$ and two $10 \mathrm{~mL}$ thin liquid (ID- 
DSI Level 0) boluses were a 50/50 mixture of E-Z-HD Barium Sulfate Powder for Suspension and the viscosity of the boluses were carefully controlled through constant temperature and periodic rechecks with a Brookfield viscometer.

\section{Experimental Procedures}

To obtain the duration, distance, and velocity of hyoid excursion, temporal and biomechanical measurements of swallowing were performed as follows.

\section{Procedures of Temporal Measurement of Hyoid Excursion (Duration)}

Each subject swallowed two $5 \mathrm{~mL}$ and two $10 \mathrm{~mL}$ liquid boluses and a total of 156 swallows were submitted for analysis. For each swallow analyzed, two picture frames were selected of the VFSE: the first showed the hyoid at resting position, and the second showed the hyoid at maximum displacement during the swallow.

Temporal measurements were taken to determine the time that elapsed between the hyoid in its resting position and in its maximum position. Before analyzing, the examiner was trained in temporal measurements. Using Adobe Premier Pro C3, swallows were looked at multiple times as whole swallows and frame-by-frame. The resting position frame was determined as the frame before the hyoid begins moving in any forward or upward motion. The maximum frame was determined as the last frame where the hyoid moved in any forward or upward motion. Adobe Premiere Pro C3 was used to determine each frame which is represented in seconds and that time was recorded into an excel sheet. After determining the time of the resting position frame of the hyoid and the time of the maximum position frame of the hyoid, the following equation was used to determine the time that elapses between the two positions:

Duration of maximum hyoid excursion = Time of maximum position - time of resting position

\section{Procedures of Biomechanical Measurement of Hyoid Excursion (Distance)}

The biomechanical measurements in this study were collected for prior study at the Swallowing Research Laboratory at Ohio University [9]. The two biomechanical displacements of hyoid excursion in this study were vertical and anterior displacements. The maximal distance of hyolaryngeal excursion was calculated using these two biomechanical measure- ments. The vertical and anterior displacements were measured as follows.

In order to accurately analyze the hyoid displacements, the VFSE S-VHS tapes were digitized using Adobe Premiere Pro C3 and a Sony DVMC-DA1 Media Converter. For each swallow analyzed, two picture frames were selected of the VFSE: the first showed the hyoid at resting position, and the second showed the hyoid at maximum displacement during the swallow. These picture frames were then submitted to the ImageJ $1.32 \mathrm{j}$ program. The resting and maximum frames of the hyoid were selected only after multiple observations of the whole swallow in real time and after frame-by-frame inspection. The resting position of the hyoid was marked as the position the hyoid was in the moment just before the bolus is propelled into the pharynx from the oral cavity. The biomechanical measures are as follows:

Each individual picture frame was then rotated to a true vertical $/ 90^{\circ}$ angle. A line between the front-bottom corner of the cervical vertebrae 4 (C4) and the front-bottom corner of $\mathrm{C} 2$ was the image that was rotated to a $90^{\circ}$ angle. This rotation simplified the forward and upward displacement of the hyoid and assured the calculation in relation to the vertebral column. Also, the distance between the front-upper and frontbottom corners of C3 was used as the scale of $15 \mathrm{~mm}$ for reference of hyoid displacement. This measurement was relative, not absolute. Two points were marked on each frame. In the resting position frames, points were marked on (1) the frontbottom corner of C4, serving as an anchor point, and (2) any point on the hyoid. In the maximum frames, points were marked on (1) the front-bottom corner of C4 again and (2) the same point on the hyoid that was marked in the resting position, but while it is in maximum position. The ImageJ program calculates the values of each point on an $(x, y)$ axis. The following formula was used to measure the forward and upward displacement of the hyoid (Figure 1).

(1) forward displacement: (x2 - x1) - (C4x2 - C4x1)

(2) upward displacement: (y2 - y1) - (C4y2 - C4y1)

Where $\mathrm{xl}$ and $\mathrm{yl}$ are the starting (rest frame) coordinates of the structure of interest and $\mathrm{x} 2$ and $\mathrm{y} 2$ are the comparison image coordinates (e.g. maximum excursion coordinates). C4x1 and $\mathrm{C} 4 \mathrm{yl}$ are the coordinates of the anchor point in the rest frame, and C4x2 and C4y2 are the coordinates of the anchor point in the comparison frame [9].

Maximum distance of the hyoid excursion ${ }^{2}=$ Maximum forward displacement ${ }^{2}+$ Maximum upward displacement ${ }^{2}$ 

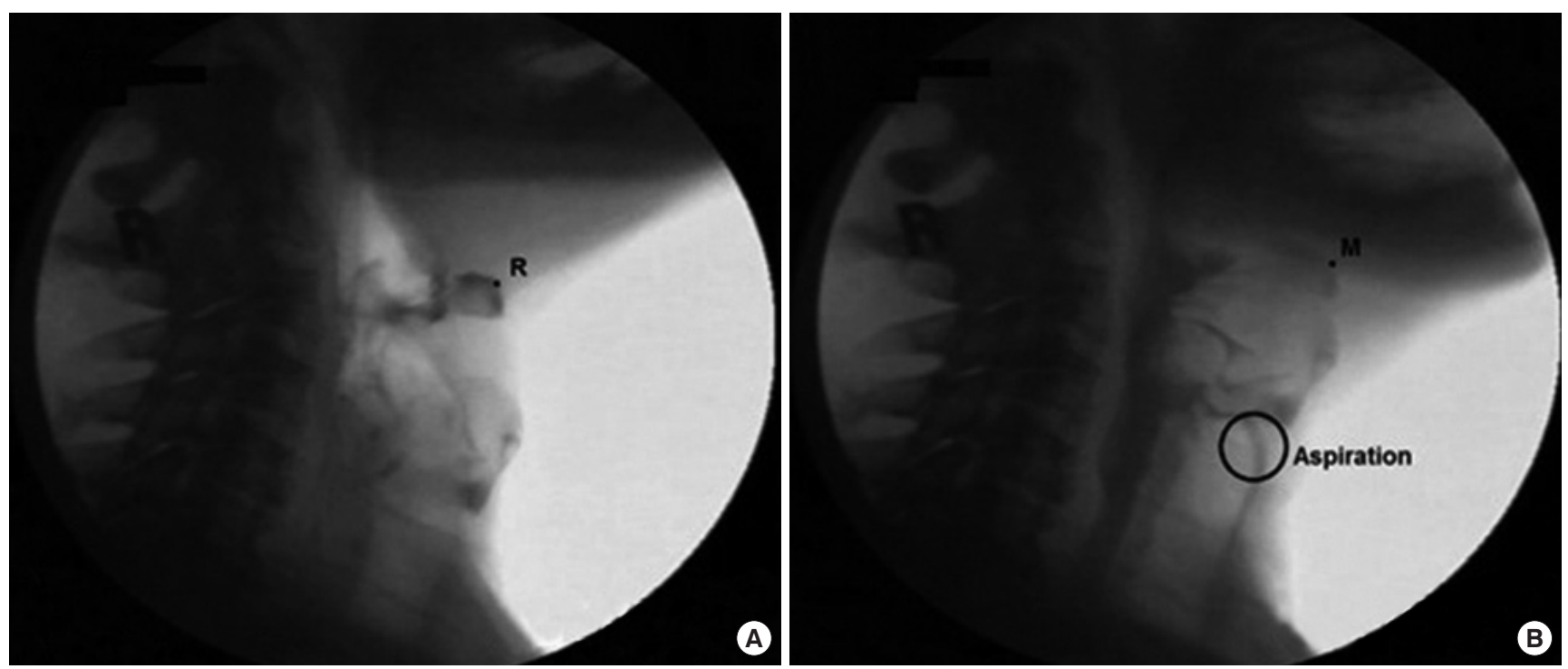

Figure 1. (A) Rest frame of hyoid bone (R), (B) Maximum displacement frame of hyoid bone during the swallowing (M) and aspiration.

\section{Procedures for Hyoid Velocity during Swallowing}

The following formula was generated to calculate the velocity of hyoid excursion:

Hyoid Excursion Velocity=Maximum distance of the hyoid $(\mathrm{cm}) /$ Duration of maximum hyoid excursion (sec)

\section{Statistical Analysis}

Group difference between aspirators and non-aspirators was analyzed for the duration, distance, and velocity of hyoid excursion during swallowing. The statistical comparisons were performed for each dependent variable using a two-way analysis of variance (ANOVA) for a repeated measure, volume of bolus as a within-subject variable, and group as between-subject variable. Wilkin's Lambda was used to determine the significance $(p<0.05)$.

\section{RESULTS}

\section{Reliability}

For inter-judge reliability, a second independent judge re-analyzed the swallows of $20 \%$ of randomly selected subjects. The second judge was a graduate student who had undergone training on temporal measures for another investigation. A significant correlation was observed $(r=0.92, p<0.01)$. For intra-judge reliability, the investigator re-analyzed the same $20 \%$ subjects a second time. A significant correlation was observed $(r=0.83, p<0.01)$. Both inter- and intra-judge reliabilities were compared using the interclass correlation coefficient
(ICC). The significance level was set at $p<0.05$.

\section{Duration of Maximum Hyoid Excursion}

The mean and confidence interval (CI) of duration time for two groups are shown in Figure 2. Aspirators displayed a mean of 1.08 seconds $(S D=0.40)$ for $5 \mathrm{~mL}$ thin liquid bolus, and a mean of 1.05 seconds $(S D=0.45)$ for $10 \mathrm{~mL}$ thin liquid bolus, respectively. Non-aspirators displayed a mean of 0.84 seconds $(S D=0.39)$ for $5 \mathrm{~mL}$, and a mean of 0.83 seconds $(S D=0.37)$ for $10 \mathrm{~mL}$, respectively. There was a significant difference in maximum hyoid excursion duration between aspirators and non-aspirators $(F(1,115)=4.39, p=0.04)$. Aspirators displayed a longer time to reach maximum hyoid excursion compared to non-aspirators.

\section{Distance of Maximum Hyoid Excursion}

The mean and CI of distance for two groups are shown in Figure 3. Aspirators displayed a mean of 2.09 centimeters $(S D=$ $0.71)$ for $5 \mathrm{~mL}$, and a mean of 2.02 centimeters $(S D=0.69)$ for $10 \mathrm{~mL}$, respectively. Non-aspirators displayed a mean of 2.22 centimeters $(S D=0.64)$ for $5 \mathrm{~mL}$, and a mean of $1.82(S D=$ 0.51 ) for $10 \mathrm{~mL}$, respectively. Aspirators moved a longer distance of hyoid excursion than non-aspirators for $5 \mathrm{~mL}$, but a shorter distance than non-aspirators for $10 \mathrm{~mL}$. There was no significant difference between the groups in relation to distance. In addition, there was no significant difference between the volumes. 


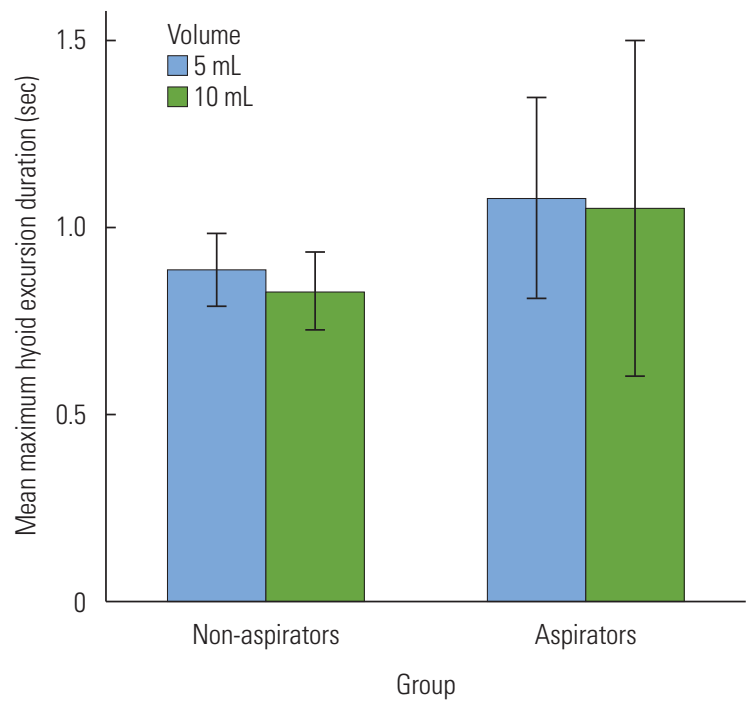

Figure 2. Mean and confidence interval (CI) of maximum duration of hyoid excursion between aspirators and non-aspirators.

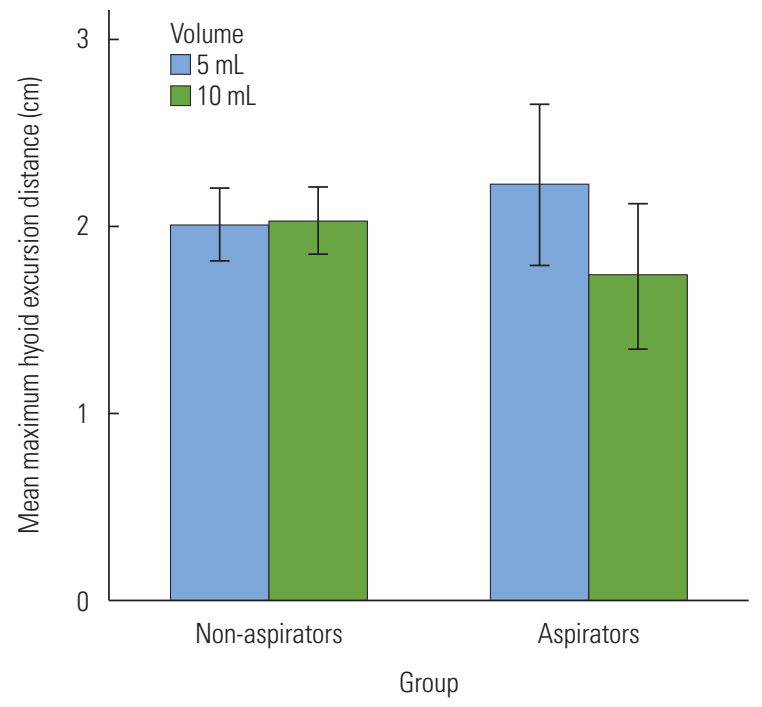

Figure 3. Mean and confidence interval (CI) of maximum distance of hyoid excursion between aspirators and non-aspirators.

\section{Velocity of Maximum Hyoid Excursion}

The mean and CI of distance for two groups are shown in Figure 4. Aspirators displayed a mean of 2.44 centimeters per second $(S D=1.30)$ for $5 \mathrm{~mL}$, and a mean of 1.82 centimeters per second $(S D=0.87)$ for $10 \mathrm{~mL}$, respectively. Non-aspirators displayed a mean of 2.72 centimeters per second $(S D=1.38)$ for $5 \mathrm{~mL}$, and a mean of 2.86 centimeters per second $(S D=$ 1.69) for $10 \mathrm{~mL}$, respectively. There was no significant difference between aspirators and non-aspirators in velocity. For both volumes, aspirators exhibited a slower velocity com-

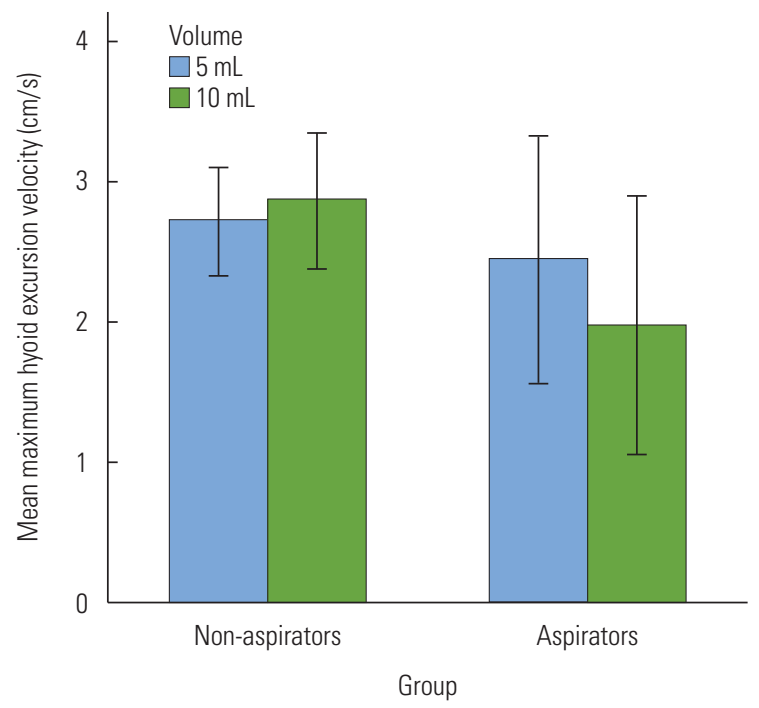

Figure 4. Mean and confidence interval (Cl) of velocity of hyoid excursion between aspirators and non-aspirators.

pared to non-aspirators. The difference between two volumes was also non-significant.

\section{DISCUSSION}

The specific aim of this study was to examine whether duration, distance, or velocity of hyoid excursion was associated with aspiration during the swallow in post-stroke survivors. The study also aimed to examine whether the volume of the bolus ( $5 \mathrm{~mL}$ or $10 \mathrm{~mL}$ ) had significant effects on duration, distance, or velocity of hyolaryngeal excursion in stroke survivors. The major finding of this study was that aspirators had a significantly longer duration time during hyoid excursion when compared to non-aspirators. Statistically, distance and overall velocity was not significant. The results of this study along with the clinical implications of the effects are discussed below.

\section{Duration of Maximum Hyoid Excursion}

According to the results of this study, aspirators displayed a longer time to reach maximum hyoid excursion compared to non-aspirators. For a swallow to be successful in protecting the airway and allowing the bolus to enter the esophagus, the actions of a swallow need to happen at an appropriate timing. Aspiration could be caused by increased duration of hyoid excursion because the epiglottis is not sealing the larynx quickly enough. Also, the UES is not opening in time, causing the bolus to pile up and get caught in the pyriform sinus surround- 
ing the esophagus. Both factors contribute to aspiration because the bolus is penetrating toward the true vocal folds before closure of the larynx is completed, and because remnants of the bolus can enter the larynx after the swallow because the bolus in its entirety did not go down the esophagus. Kendall and Leonard [11] reported that the time required for the hyoid to reach maximal elevation was prolonged in patients with dysphagia relative to the younger control group. These findings are consistent with the findings of this study. A likely explanation for longer hyoid duration in aspirators is because they attempted to compensate for a delayed initiation of pharyngeal swallowing in order to increase laryngeal closure. However, they failed to protect their airway. Karnell and Rogus [12] noted that delayed initiation of pharyngeal swallow has been described as one of the most common causes of aspiration. Kim, McCullough and Asp [13] reported stroke survivors with aspiration showed delayed pharyngeal swallow for more than one second compared to non-aspirating stroke survivors and normal subjects. For aspirators, long after the head of the bolus enters the pharynx, the pharyngeal swallow has been triggered. Similarly, Kendall and Leonard [11] also reported that the onset of hyoid elevation relative to the onset of bolus pharyngeal transit was delayed in patients with dysphagia compared to the younger control subjects. Delays and prolonged duration in pharyngeal swallows may have a significant effect on certain individuals due to impaired sensitivity and coordination of their mechanisms [12]. Stroke may have an effect on these mechanisms, therefore a prolonged hyolaryngeal excursion in stroke survivors can cause significant dysphagia sign, such as aspiration.

Normal and safe swallowing requires the coordination of many neuromuscular structures within the head and neck, combined with functioning anatomy and physiology. A stroke causing damage in the brain heavily affects its ability to direct nerve signals and muscles to operate correctly, causing dysphagia. Certain muscles connecting to the hyoid assist in its elevation, furthermore opening and widening the esophagus during swallowing. Submental muscles play a role in the upward elevation of the hyoid. Damage to these muscles caused by stroke, or disruption of signals telling these muscles when to contract has a major effect on presence of penetration and aspiration. Feng et al. [14] discovered that geniohyoid muscle atrophy associated with aging may increase risk of aspiration in older adults. They also noted that age-related loss of muscle mass and strength could contribute to a change in the position of the hyoid bone, impairing the extent of hyoid excursion needed for airway protection. Aging stroke survivors may have a disadvantage of muscular strength during the swallow.

\section{Distance of Maximum Hyoid Excursion}

The results of this study revealed the distance of hyoid excursion between aspirators and non-aspirators was not significant. This finding was consistent with other hyoid excursion measurements of swallowing conducted by Kim and McCullough [9]. They reported that there was no significant difference between aspirators and non-aspirators for both vertical and anterior displacement. Similarly, a study conducted by Steele et al. [15] on the relationship between hyoid and laryngeal displacement and swallowing impairment discovered there was no significant difference in anatomically-scaled displacement between those swallows exhibiting penetrationaspiration and no penetration-aspiration. Both studies supported the evidence that distance of hyolaryngeal excursion does not have an independently active role in the facilitation of aspiration.

\section{Velocity of Maximum Hyoid Excursion}

There was no significant difference between aspirators and non-aspirators for velocity. For both volumes, aspirators exhibited a slower velocity compared to non-aspirators. However, the reduced velocity was not significant. Although research on hyoid velocity in stroke survivors is limited, these findings can relate to previous studies involving normal swallows. A recent study by Ragland, Park, and Kim [16] revealed that age has a significant effect on the velocity of hyolaryngeal excursion. This finding could be consistent with the results of this research, seeing as most subjects who suffered stroke were above age 50 . Results concluded that velocity did not have an active role in the facilitation of aspiration, but signs of slower velocity in aspirators, although insignificant, could suggest that slower velocity in combination with stroke and older age could lead to the possibility of aspiration. This finding would provide clinicians with risk factors to check and exclude. Future studies should investigate the incidence of aspiration related to age and hyoid velocity.

\section{Bolus Volumes on Hyoid Excursion}

In relation to bolus volumes, aspirators exhibited a slower velocity for $10 \mathrm{~mL}$ compared to $5 \mathrm{~mL}$ boluses. Ueda et al. [17] reported hyoid velocity increases with increasing bolus volume. They examined the effect of bolus volume on hyoid velocity in normal subjects and results showed that maximum hyoid ve- 
locities are affected by bolus volumes. This suggests that the muscles that implement hyoid movement must contract faster as the bolus volume increases [17]. The findings of this study could reveal a link between slower contractions of oropharyngeal muscles and aspiration.

\section{Clinical Implications}

The results of this study provide clinicians with a basis of what to look for in stroke survivors at risk for aspiration. Although significant differences were not found between aspirators and non-aspirators for hyoid excursion distance and velocity, it is not correct to rule out those variables altogether. The important finding of increased duration in aspirators proves that proper timing of hyoid excursion is crucial to the safe passage of the bolus. It also provides clinicians with the opportunity to create or employ preventative measures to ensure stroke survivors do not aspirate. According to Van Der Kruis et al. [18], the supraglottic or super-supraglottic swallows would be especially useful in stroke survivors at risk of aspiration, seeing as both involve closing the airways. The supraglottic swallow involves closing the airway at the true vocal folds before and during the swallow, thus preventing aspiration during the swallow. The super-supraglottic swallow is similar in that it follows the same steps but more effort is asked of the patient. This extra effort tilts the arytenoids further forward and pulls in the false vocal folds, completely closing the entrance to the airway. Effortful swallows and Mendelsohn maneuver target directly to improve hyoid excursion for patients with reduced or prolonged excursion. Clinicians are able to implement these exercises to improve muscle strength and safety of swallowing in patients with dysphagia.

Other preventative measures include modifying bolus types. Leonard et al. [19] reported a lower aspiration rate for thickened liquid boluses compared to thin liquid boluses. Their study compared the effects of three different consistencies in patients with dysphagia. Results showed significantly less aspiration on thickened liquid than thin liquid, thus demonstrating the potential of a thicken liquid to enhance swallowing safety in patients with dysphagia. It is important to investigate the effects of bolus consistency on hyoid excursion in stroke survivors.

\section{Limitations and Future Study}

The current study consisted of only 39 subjects, which is a very small number. It is important for future studies to incorporate a higher number of subjects when collecting data in an attempt to replicate the results of the current study. Also, only one bolus consistency was used in $5 \mathrm{~mL}$ and $10 \mathrm{~mL}$ measurements for this study. Although two bolus volumes were considered in this study, different bolus consistencies should be investigated in future studies. The swallows in this study were previously recorded and some clips could not be used due to poor quality. This limited number of subjects included in the study. Future researchers are encouraged to record the swallows of their subjects to ensure quality, accuracy, and consistency.

\section{REFERENCES}

1. Hixon TJ, Weismer G, Hoit JD. Preclinical speech science: anatomy, physiology, acoustics, perception. San Diego, CA: Plural Publishing. 2008.

2. Domench E, Kelly J. Swallowing disorders. Medical Clinics of North America. 1999; 83:97-113.

3. Sheikh S, Allen E, Shell R, Hruschak J, Iram D, Castile R, McCoy K. Chronic aspiration without gastroesophageal reflux as a cause of chronic respiratory symptoms in neurologically normal infants. Chest. 2001;120:1190-1195.

4. Logemann JA. (1998). Evaluation and Treatment of Swallowing Disorders. Pro-ed: Austin, TX.

5. Dodds WJ. The physiology of swallowing. Dysphagia. 1989;3:171178.

6. Snell R. Clinical Anatomy (5th ed.). Little Brown and Company. 1995.

7. National Stroke Association. (2000). Dysphagia. Retrieved from https://www.stroke.org/we-can-help/survivors/stroke-recovery/ post-stroke-conditions/physical/dysphagia/

8. Kim Y, McCullough GY. Maximal hyoid displacement in normal swallowing. Dysphagia. 2007;23:274-279.

9. Kim Y, McCullough GY. Maximal hyoid excursion in postroke patients. Dysphagia. 2009;25:20-25.

10. McCullough GH, Wertz RT, Rosenbek JC. Sensitivity and specificity of clinical/bedside examination signs for detecting aspiration in adults subsequent to stroke. J Commun Disord. 2001;34:55-72.

11. Kendall KA, Leonard RJ. Hyoid movement during swallowing in older patients with dysphagia. Archives of Otolaryngology - Head and Neck Surgery. 2011;127: 1224-1229.

12. Karnell MP, Rogus NM. Comparison of clinician judgment and measurements of swallow response time: a preliminary report. Journal of Speech, Language, and Hearing Research. 2005;48: 1269-1279.

13. Kim Y, McCullough GH, Asp CW. Temporal measurements of pharyngeal swallowing in normal populations. Dysphagia. 2005; 20:290-296.

14. Feng X, Todd T, Hu Y, Lintzenich CR, Carr JJ, Browne JD, et al. Agerelated changes of hyoid bone position in healthy older adults with aspiration. The Laryngoscope. 2014;124: E231-E236. 
15. Steele CM, Bailey GL, Chau T, Molfenter SM, Oshalla M, Waito AA, et al. The relationship between hyoid and laryngeal displacement and swallowing impairment. Clinical Otolaryngology. 2011; 36:30-36.

16. Ragland MC, Park T, Kim Y. Age and gender difference in the velocity of hyoid excursion. Ohio Speech Hearing Association Conference, Columbus, OH. 2014.

17. Ueda N, Nohara K, Kotani Y, Tanaka N, Okuno K, Sakai T. Effects of the bolus volume on hyoid movements in normal individuals.
Journal of Oral Rehabilitation. 2013;40:491-499.

18. Van der Kruis JGJ, Baijens LWJ, Speyer R, Zwijnenberg I. Biomechanical analysis of hyoid bone displacement in videofluroscopy: a systematic review in intervention effects. Dysphagia. 2011;26:171182.

19. Leonard RJ, White C, McKenzie S, Belafsky, PC. Effects of bolus rheology on aspiration in patients with dysphagia. Journal of the Academy of Nutrition and Deities. 2014;114:590-594. 